\title{
Muons in Supernovae: Implications for the Axion-Muon Coupling
}

\author{
Robert Bollig $\odot,{ }^{1}$ William DeRocco $\odot,{ }^{2}$ Peter W. Graham $\odot,{ }^{2}$ and Hans-Thomas Janka $\oplus^{1}$ \\ ${ }^{1}$ Max-Planck-Institut für Astrophysik Karl-Schwarzschild-Straße 1, 85748 Garching, Germany \\ ${ }^{2}$ Stanford Institute for Theoretical Physics, Stanford University, Stanford, California 94305, USA
}

(Received 15 May 2020; revised 6 July 2020; accepted 15 July 2020; published 30 July 2020)

\begin{abstract}
The high temperature and electron degeneracy attained during a supernova allow for the formation of a large muon abundance within the core of the resulting protoneutron star. If new pseudoscalar degrees of freedom have large couplings to the muon, they can be produced by this muon abundance and contribute to the cooling of the star. By generating the largest collection of supernova simulations with muons to date, we show that observations of the cooling rate of SN 1987A place strong constraints on the coupling of axionlike particles to muons, limiting the coupling to $g_{a \mu}<10^{-8.1} \mathrm{GeV}^{-1}$.
\end{abstract}

DOI: 10.1103/PhysRevLett.125.051104

Introduction.-Axions are hypothetical light pseudoscalar degrees of freedom. Initially proposed to solve the strong $C P$ problem [1-3], they are a generic prediction of string theory [4] and a good candidate for dark matter [5-9]. As a result, a large research effort is dedicated to searching for these "axionlike particles" (henceforth, axions), mainly through their coupling to photons [7,10-13], electrons [14], or nucleons [15] (see [16] for a review). The coupling to muons, however, has not been well studied, partially by virtue of the fact that the high muon rest mass and short lifetime make performing precision muon experiments over long timescales difficult. Luckily, astrophysics provides us with an alternate means to probe this coupling: the cooling of supernovae. (Neutron star cooling may also place a bound, but rough estimates indicate that the supernova bound is stronger. This is a future avenue to explore.)

As noted recently [17-19], though the temperature of a supernova hardly rises above $\sim 50 \mathrm{MeV}$, there is still a large proportion of thermal photons and neutrinos with energies well above the muon rest mass. Additionally, the electrons acquire a large chemical potential allowing their conversion to muons, with the production of muon antineutrinos compensating to maintain a net zero muon number. Because of the small difference in the neutral current cross section between $\nu$ and $\bar{\nu}$, muon antineutrinos diffuse out of the protoneutron star (PNS) at a higher rate than muon neutrinos, leading to the accumulation of a net muon number within the PNS in a process known as "muonization." Recent

Published by the American Physical Society under the terms of the Creative Commons Attribution 4.0 International license. Further distribution of this work must maintain attribution to the author(s) and the published article's title, journal citation, and DOI. Funded by SCOAP ${ }^{3}$. work has shown this effect may play a non-negligible role in facilitating supernova explosions [19].

The resulting muon abundance could produce a large axion flux. Here, we exclusively consider axions with mass $m_{a} \ll \mathrm{MeV}$ and muon-dominated interactions, coupled via

$$
\mathcal{L} \subset g_{a \mu}\left(\partial_{\sigma} a\right) \bar{\psi}_{\mu} \gamma^{\sigma} \gamma_{5} \psi_{\mu} \equiv-i g_{a \mu}\left(2 m_{\mu}\right) \bar{\psi}_{\mu} \gamma_{5} \psi_{\mu} a,
$$

where $m_{\mu}$ is the muon mass, $a$ is the axion field, $\psi_{\mu}$ is the muon spinor, and $g_{a \mu}$ is the axion-muon coupling. Bounds on $g_{a \mu}$ arise from axion contributions to the muon $g-2$, at the level of $g_{a \mu} \lesssim 10^{-2.4} \mathrm{GeV}^{-1}$ [20], as well as from cosmology $[18,21,22]$ (see Supplemental Material for a detailed discussion of these limits [23]). The bounds we calculate here extend the experimentally excluded region by over 5 orders of magnitude.

Observations of SN 1987A neutrinos indicate that the resulting PNS cooled in roughly $10 \mathrm{~s}$. A new particle transferring energy more efficiently than the neutrinos would shorten this timescale, leading to the oft-cited "cooling bound" [24-30] on the axion luminosity of $L_{a} \lesssim 3 \times 10^{52} \mathrm{erg} / \mathrm{s}$. (Bar et al. [31] question this bound, but it is based on a speculative thermonuclear explosion scenario $[32,33]$ that conflicts with stellar evolution theory, supernova nucleosynthesis, and the existence of a neutron star in SN 1987A, which is strongly suggested by interpretations of recent observations by the Atacama Large Millimeter Array [34,35].)

Note that a rough estimate of this bound was made in Ref. [18], but due to its highly approximative nature, the result was subject to a large degree of uncertainty [22]. This is not unexpected, as the high muon rest mass means both the muon density and reaction rates depend sensitively on the core temperature (and muon and electron chemical potentials) of SN 1987A. In this Letter, we significantly 
TABLE I. Supernova model parameters and resulting NS baryonic (bary.) and gravitational (grav.) masses. We place our final bound with SFHo-18.8, as it produces the weakest constraint.

\begin{tabular}{lcccc}
\hline \hline Model name & $\begin{array}{c}\text { Equation } \\
\text { of state }\end{array}$ & $\begin{array}{c}\text { Progenitor } \\
\text { mass }\left(M_{\odot}\right)\end{array}$ & $\begin{array}{c}\text { NS bary. } \\
\text { mass }\left(M_{\odot}\right)\end{array}$ & $\begin{array}{c}\text { NS grav. } \\
\text { mass }\left(M_{\odot}\right)\end{array}$ \\
\hline SFHo-18.8 & SFHo [48] & $18.8[49]$ & 1.351 & 1.241 \\
SFHo-18.6 & SFHo [48] & $18.6[50]$ & 1.553 & 1.406 \\
SFHo-20.0 & SFHo [48] & $20.0[51]$ & 1.947 & 1.712 \\
LS220-20.0 & LS220 [52] & $20.0[51]$ & 1.926 & 1.707 \\
\hline \hline
\end{tabular}

improve on the previous estimate by running dedicated simulations that make use of recent results [36-44] constraining the PNS equation of state and mass, allowing us to reduce the uncertainty and set a robust bound on the coupling over 6 orders of magnitude. Interestingly, these new constraints on the equation of state (EOS) and mass lead to a generically higher core temperature, which suggests that existing bounds on other axion couplings may be strengthened by including this microphysics as well.

Profiles from simulations.-As with any supernova bound, it is critical to assess the robustness of our results to variations in the choice of model. To do this, we ran four simulations that span a range of allowable final neutron star (NS) masses for SN 1987A (see Table I). The simulations were performed in spherical symmetry (1D) with the PROMETHEUS-VERTEX code with generalrelativistic corrections and six-species neutrino transport, solving iteratively the two-moment equations for neutrino energy and momentum with a Boltzmann closure [45] and using the full set of neutrino processes listed in $[19,46]$. PNS convection was taken into account by a mixing-length treatment and explosions were artificially triggered a few $100 \mathrm{~ms}$ after bounce at the progenitor's $\mathrm{Fe} / \mathrm{Si}$ or $\mathrm{Si} / \mathrm{O}$ composition interface as described in [47].

The main astrophysical uncertainties are connected to the neutron star mass in SN 1987A and the equation of state at supernuclear densities. Model SFHo-18.6 has a canonical neutron star mass well within the range expected for the compact remnant in SN 1987A, while in models SFHo20.0 and LS220-20.0 the neutron star masses are near the upper edge of the expected range, and in model SFHo-18.8 the neutron star mass is at the lower edge of the allowed range [35]. The SFHo equation of state is fully compatible with all current constraints from nuclear theory and experiment [36-38] and astrophysics, including pulsar mass measurements [39-41] and the radius constraints deduced from gravitational-wave and Neutron Star Interior Composition Explorer measurements [42-44]. Results for the long-used LS220 equation of state are shown for reference and comparison. Note that these equations of state are considerably softer than those of previous works on axion emission from supernovae [53-55], which employed stiff equations of state that are increasingly

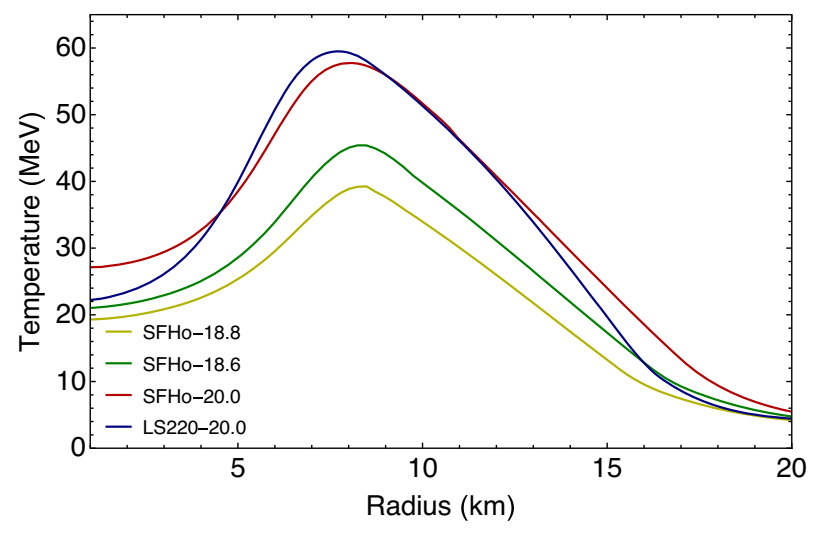

FIG. 1. Temperature profile for various models at $1 \mathrm{~s}$ postbounce.

disfavored by the constraints above. The adoption of softer equations of state generically results in a smaller radius and higher temperature than in PNS models with stiff equations of state. It should be noted that, though this change is most relevant for the axion-muon coupling, the generically higher temperatures will influence many existing supernova limits on new particles, and these results should be revisited with the new EOS constraints in mind.

We have plotted the temperature, density, and muon number density for these simulations at $1 \mathrm{~s}$ postbounce in Figs. 1-3 respectively. Note that, though the temperature varies by $30 \%$, the ultimate muon density, the quantity to which our bound is most sensitive, does not change by more than an $\mathcal{O}(1)$ factor in regions of interest, demonstrating a considerable robustness to large changes in initial parameters. In order to place conservative limits, we ultimately adopt the SFHo- 18.8 result as our fiducial profile, which is the coolest and results in the weakest constraint. Additionally, though we place our bound at $t=1 \mathrm{~s}$, we have also computed the bound at $t=0.5$ and $t=3 \mathrm{~s}$, which span the time interval of highest temperature and neutrino luminosity, and find that the ultimate limit on $g_{a \mu}$ does not change by more than a factor of 2 .

Axion production by muons.-There are two dominant contributions to the axion emissivity due to muons in the supernova: Compton scattering $(\gamma+\mu \rightarrow a+\mu)$ and

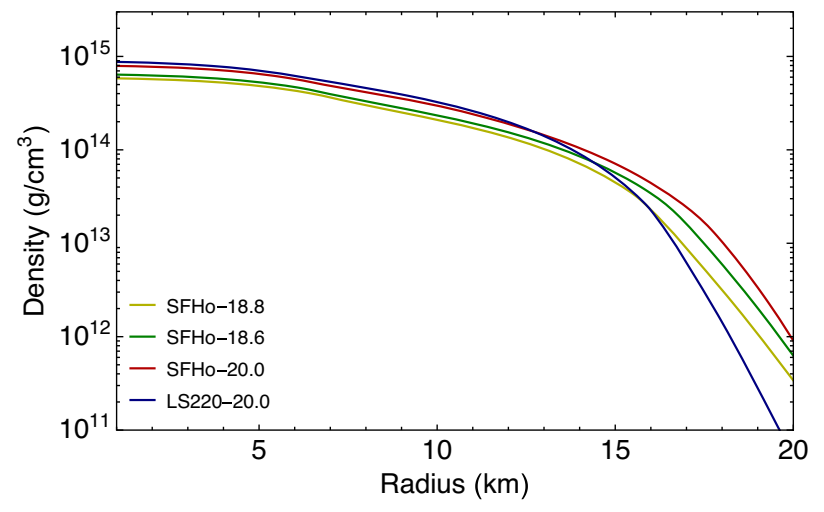

FIG. 2. Density profile for the models corresponding to Fig. 1. 


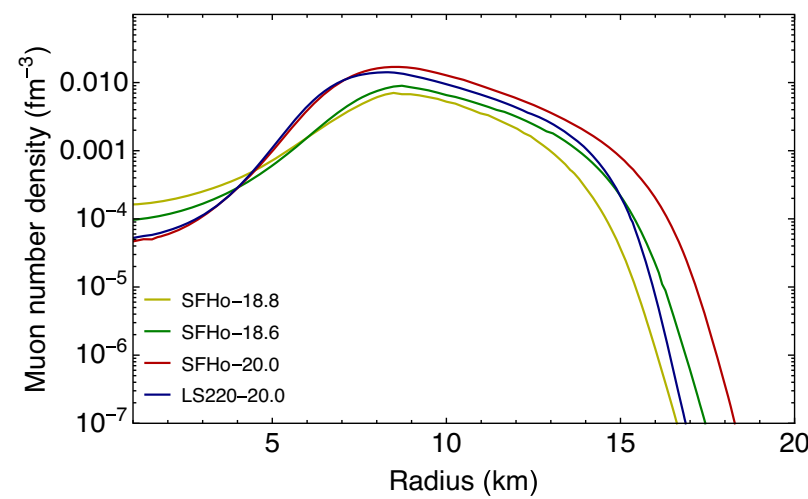

FIG. 3. Muon number density. Note that despite large differences in peak temperature, the muon number density does not change by more than an order of magnitude in the relevant region of high muon density $(5 \lesssim r \lesssim 15 \mathrm{~km}$ ).

muon-proton bremsstrahlung $(\mu+p \rightarrow \mu+p+a)$. Contributions from muon-muon bremsstrahlung are subdominant, as the muon number density is over an order of magnitude below the proton density, muon-electron bremsstrahlung is suppressed by the muon-electron mass ratio, and other channels such as Primakoff or nuclear bremsstrahlung require additional couplings of the axion.

While electrons in the core of the PNS are highly degenerate, suppressing the Compton process [56] and resulting in bremsstrahlung [24] as the dominant axionproduction channel, muons are only mildly degenerate. This reduces the suppression considerably, allowing the Compton process to become the dominant contribution to axion production. This mild degeneracy is displayed in Fig. 4, where we plot the ratio $\mu_{\mu} / T$ as a function of radius for the various profiles considered. Axion production is only effective in regions of high muon density $(5 \lesssim r \lesssim 15 \mathrm{~km})$, where we observe that the degeneracy parameter remains a small $\mathcal{O}(1)$ value.

In order to quantify the effects of the degeneracy on the Compton rate, we compute the approximate suppression factor $F_{\text {deg }}$ by averaging over the Pauli blocking factor (see Sec. 3.2.5 of Ref. [24]),

$$
F_{\mathrm{deg}} \equiv \frac{1}{n_{\mu}^{\mathrm{th}}} \int \frac{2 d^{3} \mathbf{p}}{(2 \pi)^{3}} f_{\mu}(E)\left[1-f_{\mu}(E)\right]
$$

where $n_{\mu}^{\text {th }}$ is the thermal number density and $f_{\mu}(E)=$ $\left(e^{\left(E-\mu_{\mu}\right) / T}+1\right)^{-1}$ is the energy distribution of muons at the given degeneracy and temperature. Because of the mild degeneracy, this suppression is never in excess of $\sim 15 \%$ (see Supplemental Material for a plot of $F_{\text {deg }}$ as a function of radius [23]).

We also compute the degeneracy parameter for the protons, as previous work has suggested that proton degeneracy can play a significant role in the core [57], but find that protons are solely degenerate for $r \lesssim 4 \mathrm{~km}$,

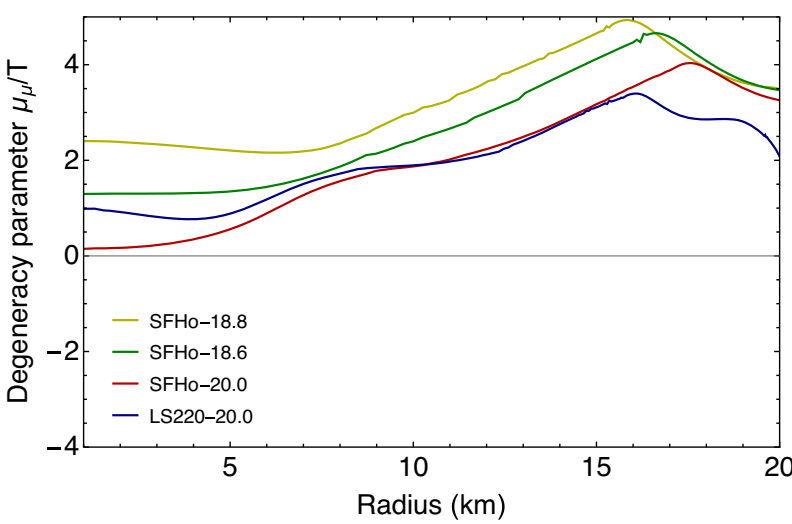

FIG. 4. Muon degeneracy parameter $\mu_{\mu} / T$ for various profiles. This ratio never exceeds a small $\mathcal{O}(1)$ value in the relevant region of high muon density $(5 \lesssim r \lesssim 15 \mathrm{~km})$.

which is outside the region of high muon abundance; hence, proton degeneracy effects are neglected.

Given the above results and the nonrelativistic velocities of the muons, the production rate for the Compton process is [58]

$$
\Gamma^{\text {Compton }}=\frac{\alpha\left(2 g_{a \mu} m_{\mu}\right)^{2} \omega^{2}}{3 m_{\mu}^{4}} \frac{n_{\mu}}{e^{\omega / T}-1} F_{\mathrm{deg}}
$$

with $\omega$ as the energy of the emitted axion. For muon-proton bremsstrahlung, we have [58]

$\Gamma^{\text {brem }}=\alpha^{2}\left(2 g_{a \mu} m_{\mu}\right)^{2} \frac{8 \pi}{3 \sqrt{2 \pi}} \frac{n_{p} n_{\mu}}{\sqrt{T} m_{\mu}^{7 / 2} \omega} e^{-\omega / T} F(w, y)$

with

$$
F(w, y)=\int_{0}^{\infty} d x x e^{-x^{2}} \int_{\sqrt{x^{2}+w}-x}^{\sqrt{x^{2}+w}+x} \frac{t^{3}}{\left(t^{2}+y^{2}\right)^{2}} d t
$$

where we have defined $w \equiv \omega / T$ and $y \equiv k_{S} / \sqrt{2 m_{\mu} T}$ with $k_{S}$ as the Debye screening scale, which is the appropriate scale to use for nondegenerate conditions.

There are two relevant regimes of the $g_{a \mu}$ parameter space: the "free-streaming regime" (small $g_{a \mu}$ ) and the "trapping regime" (large $g_{a \mu}$ ). In the free-streaming regime, the mean free path for axion absorption is significantly larger than the scale of the PNS; hence, axions can escape the inner regions of the star without any further interactions, leading to volume emission. At sufficiently strong couplings, however, we enter the trapping regime. The mean free path becomes small in the interior of the PNS and axions are rapidly produced and reabsorbed out to some radius where the mean free path grows long and they escape. This radius is known as the "axion sphere" [59] and the luminosity can be approximated by blackbody emission from that radius at the local temperature [24]. 
Let us begin with the free-streaming regime. In order to compute the free-streaming luminosity of axions due to the muon coupling, we use the following [28]:

$$
\begin{aligned}
L_{a}^{\text {free }}= & \int d V \int \frac{\left(4 \pi \omega^{2}\right) d \omega}{(2 \pi)^{3}} e^{-\tau(\omega, r)} \omega\left(\Gamma^{\text {Compton }}(\omega, r)\right. \\
& \left.+\Gamma^{\operatorname{brem}}(\omega, r)\right),
\end{aligned}
$$

with $\tau$ the optical depth, given by

$\tau(\omega, r)=\int_{r}^{\infty} d r^{\prime}\left(\Gamma^{\text {Compton }}(\omega, r)+\Gamma^{\text {brem }}(\omega, r)\right) e^{\omega / T}$,

where the factor of $e^{\omega / T}$ appears since we are now considering the absorptive widths of the processes, which are related to the production rates by detailed balance: $\Gamma_{\text {prod }}=e^{-\omega / T} \Gamma_{\text {abs }}$. We include the factor of $e^{-\tau(\omega, r)}$ in the computation of the luminosity to account for the moderate reabsorption of axions by muons even in the free-streaming regime. [An early version of this Letter contained an additional factor of $2 \omega$ in the denominator of Eq. (6), which was in error. This caused a factor of 100 suppression in our bounds. We thank the authors of Ref. [60] for bringing this to our attention.]

In the trapped regime, we must first identify the axion sphere, which is done by computing the Rosseland mean opacity for axions, given by

$$
\frac{1}{\rho \kappa_{a}}=\frac{15}{8 \pi^{4}} \int_{0}^{\infty} d x\left(\Gamma_{\mathrm{abs}}^{\text {Compton }}+\Gamma_{\mathrm{abs}}^{\mathrm{brem}}\right)^{-1} \frac{x^{4} e^{2 x}}{\left(e^{x}-1\right)^{3}},
$$

where $x \equiv \omega / T$. We can then integrate this radially to find the optical depth $\tau_{R}$,

$$
\tau_{R}(r)=\int_{r}^{\infty} \rho \kappa_{a} d r
$$

Whenever $\tau_{R} \gg 1$, the axions are efficiently trapped [30]. The axion sphere is defined as the radius $r_{a}$ at which $\tau_{R}\left(r_{a}\right)=\frac{2}{3}$. The resulting luminosity is then just approximated as blackbody emission from that radius,

$$
L_{a}^{\text {trapped }}=\frac{\pi^{2}}{120}\left(4 \pi r_{a}^{2}\right) T^{4} .
$$

As this approximation implicitly assumes that all axions decouple at the same radius, it is only applicable in regions where the coupling is strong enough that absorptive processes shut off over a very narrow region. Otherwise, the energy dependence of the absorptive width stretches this single radius of decoupling into an extended decoupling region. At large values of the coupling, the approximation of an axion sphere is applicable. At the lower limit, as we will show below, the trapped regime transitions smoothly into the free-streaming regime while still

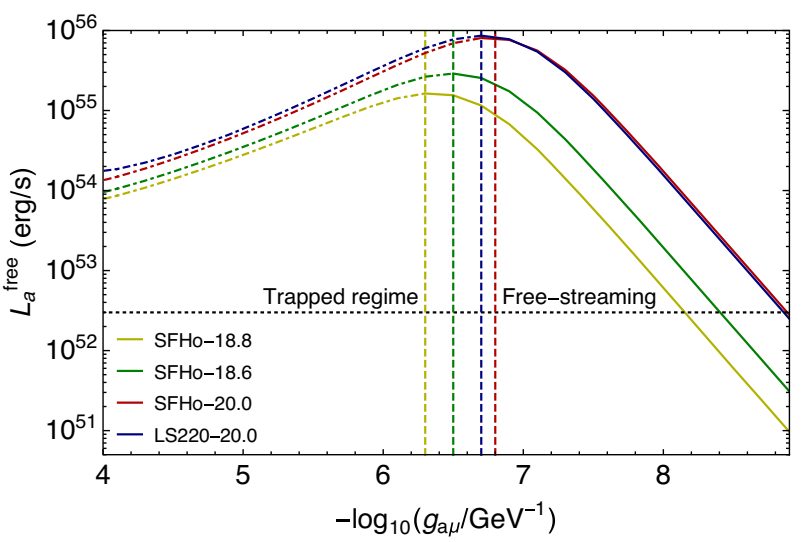

FIG. 5. The free-streaming luminosity in axions as a function of axion-muon coupling. Couplings for which the curves lie above the dotted black line and to the right of the dashed lines are excluded by the free-streaming luminosity, while couplings to the left of the vertical dashed lines are excluded by the blackbody approximation in the trapped regime. The decrease of the freestreaming luminosity toward weak couplings is due to rapidly declining axion production, whereas the decrease toward strong couplings is due to an increasing absorptive width and corresponding suppression by the $e^{-\tau(\omega, r)}$ term. Note that the free-streaming luminosity is not the total luminosity, as it neglects the contribution from blackbody surface emission when the optical depth is large. It is shown to the left of the vertical lines purely to demonstrate the expected falloff in the free-streaming (volume) emission and has hence been plotted as dot-dashed lines in that region.

producing luminosities in excess of the cooling constraint; hence, the effect of an extended decoupling region does not affect our overall bound.

Results.-Figure 5 shows the results of our luminosity calculations. The curves correspond to the free-streaming luminosity given in Eq. (6) as a function of coupling, with the horizontal axis showing $-\log _{10}\left(g_{a \mu} / \mathrm{GeV}^{-1}\right)$. Note that, toward weak couplings, the luminosity falls off as the coupling becomes too weak to allow the efficient production of axions. Toward stronger couplings, the freestreaming luminosity also falls off, now due to the increasing absorptive width (i.e., the factor of $e^{-\tau(\omega, r)}$ causes a large suppression).

The vertical lines demarcate our free-streaming regime from our trapped regime. We separate these two regimes at the coupling for which the optical depth within the axion sphere rises above $2 / 3$. Note that the transition occurs at couplings where we are still excluded by free streaming, allowing a smooth transition between regimes and no breaks in our bounds. If, instead, the trapped regime had only begun at much stronger couplings, there could have, in principle, been a gap in the constraint between the two regimes. But this is far from the considered case; hence, the actual details of the transition between regimes do not affect our overall results. Note that, even at high couplings, the axion sphere is never further out than $\approx 18 \mathrm{~km}$, as should be evident from the plot of the muon number density 


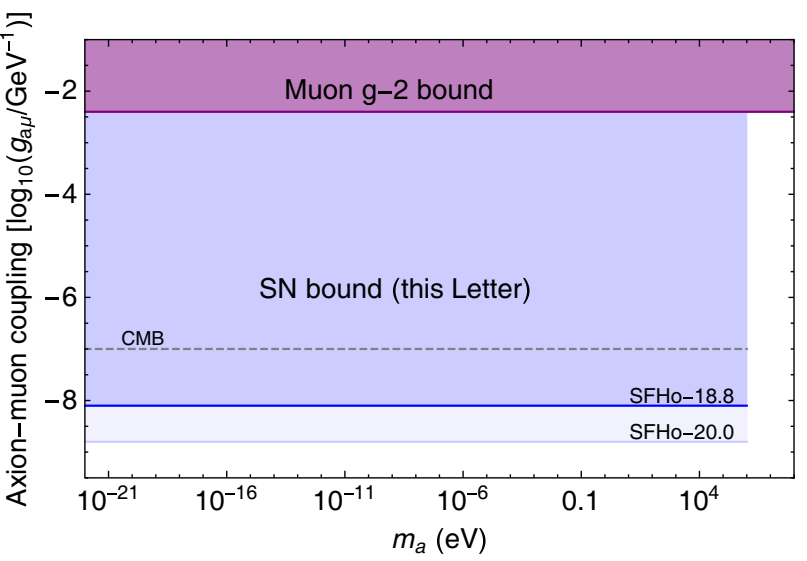

FIG. 6. Our constraints, plotted on axion parameter space. Excluded regions are colored. We show the bound that is placed with the SFHo-18.8 profile as a solid blue line, which we quote as our ultimate bound. Note that this is extremely conservative, as model SFHo-18.8 is on the low edge of those compatible with SN 1987A. To illustrate the model dependence, we also show the result for SFHo-20.0 (light blue), which sits on the high edge of the allowed range. We additionally plot existing bounds from virtual axion contributions to the muon $g-2$ (purple), which are the most robust experimental constraints [20], and a rough estimate of constraints from the Cosmic Microwave Background (CMB) (dashed line, see Supplemental Material for a thorough discussion [23]).

(Fig. 3). An axion sphere at $18 \mathrm{~km}$ corresponds to a blackbody luminosity of $\gtrsim 10^{53} \mathrm{erg} / \mathrm{s}$, so our bounds extend smoothly to high couplings.

In summary, we can exclude all couplings to the left of the vertical lines (trapped regime) and can exclude all couplings to the right of the vertical line for which the freestreaming luminosity lies above the horizontal line corresponding to $L_{a}=3 \times 10^{52} \mathrm{erg} / \mathrm{s}$ (free-streaming regime).

These results can be reformulated into a constraint on the axion coupling, the result of which is displayed in Fig. 6. Translated into a numerical bound, the constraint from SFHo-18.8 is $g_{a \mu}<10^{-8.1} \mathrm{GeV}^{-1}$. Note that this is a conservative constraint, as it is placed with the coolest profile, a profile that is on the low-mass edge of the allowed range for SN 1987A's remnant. As such, we also show the bound from SFHo-20.0 (lighter blue), which sits on the high-mass edge of the allowed range, though we only quote the conservative bound as our final result.

Additionally, we have cut off our bound at $m_{a}=1 \mathrm{MeV}$ as our production calculations implicitly took the axion to be effectively massless, a good approximation when $m_{a} \ll T$, as is the case here. More parameter space (up to $m_{a} \sim 100 \mathrm{MeV}$ ) could be excluded with a full treatment of a massive axion, though the corresponding Boltzmann suppression will cause the bounds to rise rapidly toward higher masses (see Ref. [60]).

In conclusion, supernova cooling constrains the axionmuon coupling to

$$
g_{a \mu}<10^{-8.1} \mathrm{GeV}^{-1}
$$

for axions with masses less than a $\mathrm{MeV}$, severely limiting the parameter space for exotic axionlike particles with muon-dominated interactions.

While this analysis was performed in the context of one specific model (an axion with muon-dominated interactions), we wish to note that the general insights presented here open up supernovae as a promising new avenue for robustly probing muonic beyond the Standard Model physics. (For one such extension of this Letter, see, e.g., [60].)

The full profile data for the supernova simulations used here can be found at the Garching Core-Collapse Supernova Archive [61].

The authors would like to express our deep appreciation to Djuna Croon, Gilly Elor, Rebecca Leane, and Sam McDermott for alerting us to the mistake in our Eq. (6) and the subsequently calculated limits. We thank them for reaching out prior to the publication of their paper [60], which significantly extends our work to other couplings and models. Additionally, we would like to thank Ben Wallisch and Dan Green for very useful discussions on the CMB bounds. W. D. and P. W. G. would like to express their gratitude for the support provided by DOE Award No. DE-SC0012012, by NSF Grant No. PHY-1720397, the Heising-Simons Foundation Grants No. 2015-037 and No. 2018-0765, DOE HEP QuantISED Award No. 100495, and the Gordon and Betty Moore Foundation Grant No. GBMF7946. R. B. and H. T. J. acknowledge funding by the European Research Council through Grant ERC-AdG No. 341157-COCO2CASA and by the Deutsche Forschungsgemeinschaft (DFG, German Research Foundation) through Sonderforschungsbereich (Collaborative Research Centre) SFB-1258 "Neutrinos and Dark Matter in Astro- and Particle Physics (NDM)" and under Germany's Excellence Strategy through Cluster of Excellence ORIGINS (EXC-2094)-390783311.

[1] R. D. Peccei and H. R. Quinn, Phys. Rev. Lett. 38, 1440 (1977).

[2] R. D. Peccei and H. R. Quinn, Phys. Rev. D 16, 1791 (1977).

[3] S. Weinberg, Phys. Rev. Lett. 40, 223 (1978).

[4] A. Arvanitaki, S. Dimopoulos, S. Dubovsky, N. Kaloper, and J. March-Russell, Phys. Rev. D 81, 123530 (2010).

[5] J. Preskill, M. B. Wise, and F. Wilczek, Phys. Lett. B 120, 127 (1983).

[6] L. Abbott and P. Sikivie, Phys. Lett. B 120, 133 (1983).

[7] M. Dine and W. Fischler, Phys. Lett. B 120, 137 (1983).

[8] L. D. Duffy and K. van Bibber, New J. Phys. 11, 105008 (2009).

[9] A. Ringwald, Proc. Sci., NOW2016 (2016) 081 [arXiv: 1612.08933]. 
[10] V. Anastassopoulos et al. (CAST Collaboration), Nat. Phys. 13, 584 (2017).

[11] N. Du, N. Force, R. Khatiwada, E. Lentz, R. Ottens, L. J. Rosenberg, G. Rybka, G. Carosi, N. Woollett, D. Bowring et al. (ADMX Collaboration), Phys. Rev. Lett. 120, 151301 (2018).

[12] B. M. Brubaker et al., Phys. Rev. Lett. 118, 061302 (2017).

[13] M. Betz, F. Caspers, M. Gasior, M. Thumm, and S. W. Rieger, Phys. Rev. D 88, 075014 (2013).

[14] K. Barth, A. Belov, B. Beltran, H. Bräuninger, J. Carmona, J. Collar, T. Dafni, M. Davenport, L. D. Lella, C. Eleftheriadis et al., J. Cosmol. Astropart. Phys. 05 (2013) 010.

[15] D. F. J. Kimball et al., arXiv:1711.08999.

[16] P. W. Graham, I. G. Irastorza, S. K. Lamoreaux, A. Lindner, and K. A. van Bibber, Annu. Rev. Nucl. Part. Sci. 65, 485 (2015).

[17] A. Lohs, G. Martinez-Pinedo, and T. Fischer, Proc. Sci., NICXIII2015 (2015) 118.

[18] C. Brust, D. E. Kaplan, and M. T. Walters, J. High Energy Phys. 12 (2013) 058.

[19] R. Bollig, H. T. Janka, A. Lohs, G. Martinez-Pinedo, C. J. Horowitz, and T. Melson, Phys. Rev. Lett. 119, 242702 (2017).

[20] S. Andreas, O. Lebedev, S. Ramos-Sanchez, and A. Ringwald, J. High Energy Phys. 08 (2010) 003.

[21] D. Baumann, D. Green, and B. Wallisch, Phys. Rev. Lett. 117, 171301 (2016).

[22] F. D'Eramo, R. Z. Ferreira, A. Notari, and J. L. Bernal, J. Cosmol. Astropart. Phys. 11 (2018) 014.

[23] See Supplemental Material at http://link.aps.org/ supplemental/10.1103/PhysRevLett.125.051104 for additional plots and a discussion of the CMB bounds.

[24] G. G. Raffelt, Stars as Laboratories for Fundamental Physics (University of Chicago Press, Chicago, 1996), ISBN 9780226702728.

[25] M. S. Turner, Phys. Rev. Lett. 60, 1797 (1988).

[26] A. Burrows, M. S. Turner, and R. P. Brinkmann, Phys. Rev. D 39, 1020 (1989).

[27] G. G. Raffelt, Phys. Rep. 198, 1 (1990).

[28] J. H. Chang, R. Essig, and S. D. McDermott, J. High Energy Phys. 09 (2018) 051.

[29] W. DeRocco, P. W. Graham, D. Kasen, G. Marques-Tavares, and S. Rajendran, Phys. Rev. D 100, 075018 (2019).

[30] P. Carenza, T. Fischer, M. Giannotti, G. Guo, G. MartinezPinedo, and A. Mirizzi, J. Cosmol. Astropart. Phys. 10 (2019) 016.

[31] N. Bar, K. Blum, and G. D’Amico, Phys. Rev. D 101, 123025 (2020).

[32] K. Blum and D. Kushnir, Astrophys. J. 828, 31 (2016).

[33] D. Kushnir and B. Katz, Astrophys. J. 811, 97 (2015).

[34] P. Cigan, M. Matsuura, H. L. Gomez, R. Indebetouw, F. Abellán, M. Gabler, A. Richards, D. Alp, T. A. Davis, H.-T. Janka et al., Astrophys. J. 886, 51 (2019).
[35] D. Page, M. V. Beznogov, I. Garibay, J. M. Lattimer, M. Prakash, and H.-T. Janka, arXiv:2004.06078.

[36] T. Fischer, M. Hempel, I. Sagert, Y. Suwa, and J. SchaffnerBielich, Eur. Phys. J. A 50, 46 (2014).

[37] M. Oertel, M. Hempel, T. Klähn, and S. Typel, Rev. Mod. Phys. 89, 015007 (2017).

[38] T. Fischer, N.-U. Bastian, D. Blaschke, M. Cierniak, M. Hempel, T. Klähn, G. Martínez-Pinedo, W. G. Newton, G. Röpke, and S. Typel, Pub. Astron. Soc. Aust. 34, e067 (2017).

[39] P. B. Demorest, T. Pennucci, S. M. Ransom, M. S. E. Roberts, and J. W. T. Hessels, Nature (London) 467, 1081 (2010).

[40] J. Antoniadis, P. C. C. Freire, N. Wex, T. M. Tauris, R. S. Lynch, M. H. van Kerkwijk, M. Kramer, C. Bassa, V. S. Dhillon, T. Driebe et al., Science 340, 1233232 (2013).

[41] H. T. Cromartie, E. Fonseca, S. M. Ransom, P. B. Demorest, Z. Arzoumanian, H. Blumer, P. R. Brook, M. E. DeCesar, T. Dolch, J. A. Ellis et al., Nat. Astron. 4, 72 (2020).

[42] B. P. Abbott et al. (LIGO Scientific and Virgo Collaborations), Phys. Rev. Lett. 121, 161101 (2018).

[43] A. Bauswein, O. Just, H.-T. Janka, and N. Stergioulas, Astrophys. J. Lett. 850, L34 (2017).

[44] R. Essick, I. Tews, P. Landry, S. Reddy, and D. E. Holz, arXiv:2004.07744.

[45] M. Rampp and H. T. Janka, Astron. Astrophys. 396, 361 (2002).

[46] H.-T. Janka, Annu. Rev. Nucl. Part. Sci. 62, 407 (2012).

[47] A. Mirizzi, I. Tamborra, H. T. Janka, N. Saviano, K. Scholberg, R. Bollig, L. Hüdepohl, and S. Chakraborty, Nuovo Cimento Riv. Ser. 39, 1 (2016).

[48] A. W. Steiner, M. Hempel, and T. Fischer, Astrophys. J. 774, 17 (2013).

[49] T. Sukhbold, S. E. Woosley, and A. Heger, Astrophys. J. 860, 93 (2018).

[50] S. E. Woosley, A. Heger, and T. A. Weaver, Rev. Mod. Phys. 74, 1015 (2002).

[51] S. E. Woosley and A. Heger, Phys. Rep. 442, 269 (2007).

[52] J. M. Lattimer and F. Swesty, Nucl. Phys. A535, 331 (1991).

[53] J. H. Chang, R. Essig, and S. D. McDermott, J. High Energy Phys. 01 (2017) 107.

[54] T. Fischer, S. Chakraborty, M. Giannotti, A. Mirizzi, A. Payez, and A. Ringwald, Phys. Rev. D 94, 085012 (2016).

[55] K. Nakazato, K. Sumiyoshi, H. Suzuki, T. Totani, H. Umeda, and S. Yamada, Astrophys. J. Suppl. 205, 2 (2013).

[56] G. G. Raffelt, Phys. Rev. D 33, 897 (1986).

[57] A. Payez, C. Evoli, T. Fischer, M. Giannotti, A. Mirizzi, and A. Ringwald, J. Cosmol. Astropart. Phys. 02 (2015) 006.

[58] J. Redondo, J. Cosmol. Astropart. Phys. 12 (2013) 008.

[59] A. Burrows, M. T. Ressell, and M. S. Turner, Phys. Rev. D 42, 3297 (1990).

[60] D. Croon, G. Elor, R. K. Leane, and S. D. McDermott, arXiv:2006.13942.

[61] https://wwwmpa.mpa-garching.mpg.de/ccsnarchive/archive .html. 